\title{
Mapas na Web
}

\section{Alfredo Pereira de Queiroz Filho ${ }^{1}$ Mariana Abrantes Giannotti ${ }^{2}$}

\begin{abstract}
Resumo: Este trabalho aborda a trajetória recente dos mapas na Web. Baseia-se na constatação de que os mapas se moldaram às características da comunicação via rede, que demanda a integração e o compartilhamento das informações. A conjugação destas duas metas, no meio computacional, é denominada de interoperabilidade. Esta, é baseada em dois processos distintos: padronização e sistematização. Em decorrência da sua veiculação na Web, constata-se que o volume de mapas produzidos aumentou exponencialmente e suas funcionalidades se ampliaram. No entanto, parte dos termos relacionados aos mapas se tornou efêmera em virtude das constantes inovações oriundas do desenvolvimento tecnológico. Um dos principais desafios é tornar o conteúdo dos mapas compreensível universalmente, por homens e máquinas. As Infraestruturas de Dados Espaciais, os mapas colaborativos, a diversificação das estratégias de localização, a Digital Earth e a computação em nuvens revelam importantes tendências de desenvolvimento.
\end{abstract}

Palavras-chave: mapas; web; integração; compartilhamento; interoperabilidade; metadados; IDE; Web semântica; ontologias.

Abstract: This work deals with the recent trends of maps on the Web. It is based on the finding that the maps have been shaped to the characteristics of network communications, that requires the integration and sharing of information. The combination of these two goals, in computational vocabulary, is called interoperability. This is based on two distinct processes: standardization and systematization. On the Web, the volume of maps produced increased exponentially and its functionalities amplified. However, part of the terms related to maps become ephemeral due to constant innovations arising from technological development. One of the main challenges is to make the content of maps universally comprehensible by humans and machines. The Spatial Data Infrastructures, collaborative maps, wide range of localization strategies, digital earth and cloud computing reveal important trends of development.

Key-words: maps, web, integration, data sharing, interoperability, metadata, SDI, Semantic Web, ontologies.

\section{INTRODUÇÃO}

Elaborado para a edição comemorativa de trinta anos da Revista do Departamento de Geografia (RDG) este artigo apresenta uma abordagem sobre as transformações relacionadas aos mapas, a partir da década de 1980.

\footnotetext{
${ }^{1}$ Graduado em Geografia pela Universidade de São Paulo, mestrado em Engenharia pela Universidade de São Paulo, doutorado em Engenharia pela Universidade de São Paulo. Professor do Departamento de Geografia da Faculdade de Filosofia, Letras e Ciências Humanas da Universidade de São Paulo. E-mail: aqueiroz@usp.br

${ }^{2}$ Graduação em Engenharia Agronômica pela Universidade de São Paulo, mestrado em Sensoriamento Remoto pelo Instituto Nacional de Pesquisas Espaciais, doutorado pela Escola Politécnica da Universidade de São Paulo. Professora do Departamento de Engenharia de Transportes da Universidade de São Paulo. E-mail: mariana.giannotti@gmail.com
} 
Nesse intervalo, os periódicos, assim como os mapas, reduziram significativamente o uso do papel na veiculação dos seus conteúdos. Balzaquiana, a revista amadureceu. Renovada, incorporou o sistema Open Journal System de editoração, via Web, e interrompeu a impressão dos seus volumes em papel.

O gerenciamento e a veiculação mapas, no referido período, vivenciou processo semelhante ao das revistas acadêmicas. Em 1985, Mark S. Monmonier previu que, por causa da transição eletrônica da Cartografia, elaborar um bom mapa se tornaria tão comum quanto escrever uma boa carta.

Para Peterson (1999), o início desta dramática mudança ocorreu em meados da década de 80, com o aperfeiçoamento das interfaces dos computadores. Os progressos tecnológicos de armazenamento e comunicação de dados, como CD-ROM, DVD, Internet e Web, no início de 1990, contribuíram para a segunda grande onda de desenvolvimento cartográfico.

Este contexto e período compõem o cenário geral da pesquisa. $\mathrm{O}$ artigo apresenta uma abordagem sobre a trajetória recente dos mapas, destacando sua inserção na Web.

O argumento é de que os mapas espelham a essência da Internet. A representação se moldou ao meio no qual é veiculado. Para que sua utilização se amplie, em funcionalidades e usuários, há a necessidade de integração e compartilhamento das suas informações.

No meio computacional, integração e compartilhamento se traduzem pelo termo interoperabilidade. Esta, se caracteriza por dois principais processos: de padronização dos seus componentes e, posteriormente, o de sistematização de conceitos e termos.

Os arquivos dos mapas, como os blocos de montar (ex.: Lego), são padronizados e particionados para serem veiculados na Web. E, para que essa multiplicidade de partes seja amplamente utilizada, é necessário agrupá-la em categorias cujos significados sejam compartilhados entre seus usuários. Paralelamente, diante da dificuldade de gerenciamento do extraordinário e crescente volume de dados da rede, surge a necessidade de que os mapas sejam compreendidos univocamente por máquinas e programas computacionais.

\section{Sobre o meio e a representação}

\section{Internet ou Web?}

$\mathrm{Na}$ forma coloquial, esses termos são frequentemente usados como sinônimos. No entanto, eles são elementos distintos. A Internet se refere à infraestrutura de rede e a Web é a 
denominação de um dos serviços que utiliza a rede (PENG; TSOU, 2003). Assim, os mapas trafegam pela Internet, mas são visualizados e manipulados pelas interfaces da Web.

A Internet consiste em um sistema de redes interconectadas que utiliza o protocolo TCP/IP (Transmission Control Protocol / Internet Protocol). O protocolo TCP é responsável por dividir e remontar os dados em pacotes de informação, enquanto o protocolo IP encaminha esses pacotes desde a origem até o seu destino.

Seu sucesso está baseado em duas principais características: 1) o protocolo TCP/IP permite a comunicação entre computadores independentemente do sistema operacional utilizado (Windows, Unix, Linux, etc.), 2) não há necessidade de estabelecimento de uma conexão exclusiva de contato entre o receptor e o emissor. Os pacotes enviados seguem por várias rotas diferentes ao mesmo tempo, para depois serem remontados no local de recepção.

A sua arquitetura, denominada de cliente/servidor, pode ser definida como um modelo de interação de um sistema distribuído, onde as partes se comunicam por intermédio de perguntas e respostas. Resumidamente: o solicitante é denominado de cliente e o programa que responde é chamado de servidor. O termo servidor se aplica, genericamente, a qualquer programa que ofereça um serviço que possa ser acionado pela rede. Um servidor aceita a solicitação, executa a operação e retorna o resultado ao solicitante. O programa que está em execução torna-se um cliente quando envia uma solicitação ao servidor e espera a resposta. Segundo Fu; Sun (2011), a Internet só ganhou popularidade após 1990, quando Tim BernersLee criou um serviço para facilitar o uso da Internet. A complexidade de uso da rede, até então, restringia sua utilização às universidades, agências de pesquisa e instituições governamentais. Berners-Lee desenvolveu o primeiro servidor e o primeiro navegador da web, baseados em: Hypertext Transfer Protocol (HTTP), Hypertext Markup Language (HTML) e Uniform Resource Locator (URL), denominando-os de World Wide Web.

World Wide Web, ou simplesmente Web, é um serviço de acesso à rede Internet. É uma grande coleção de documentos hipermídia interligados por hipertextos. É um sistema distribuído de informações que utiliza o protocolo HTTP (FU; SUN, 2011). 


\section{Mapas na Web}

Mapa é um termo polissêmico. De acordo com Andrews (1996), dentre as 321 definições para a palavra mapa, extraídas de dicionários, glossários, enciclopédias, textos, jornais e monografias do período de 1649 a 1996, destaca-se a ideia de representação.

Essa constatação é ratificada por um considerável número de instituições e pesquisadores. Para a International Cartography Association (1973), mapa é uma representação da Terra ou de qualquer corpo celestial. Para Robinson et al (1995), mapa é uma representação gráfica das configurações geográficas. De forma semelhante, Muehrcke et al (2001) definem mapa como uma representação espacial do ambiente.

A adoção de protocolos de comunicação comuns e de formatos de arquivos padronizados de mapas, induzida pela ascensão da Web, ampliou os tradicionais atributos dos mapas. De acordo com MacEachren; Kraak (2001) existe, ao menos, uma nova função para o mapa: a de interface dos bancos de dados. De acordo com os autores:

"No passado, os mapas de papel foram confeccionados para armazenar e apresentar informações. Com o advento da Cartografia Digital e dos SIGs, nos anos de 1960, esta tarefa foi dividida. Desenvolvimentos recentes estão tornando possível, novamente, a junção do armazenamento, nos bancos de dados, com a exibição, na tela, de forma nunca antes possível. O mapa é agora uma interface que, se bem construída, pode dar suporte e acesso às informações e à construção de conhecimento, além de preservar o seu tradicional papel de apresentação de dados. Num ambiente baseado em mapas, eles podem literalmente usar a World Wide Web como seu banco de dados" (MACEACHREN; KRAAK, 2001, p.4).

Em decorrência, o potencial de uso dos mapas se expandiu (ver Figura 1). Além de armazenar e apresentar informações, características tradicionais dos mapas impressos, sua versão digital viabilizou o aumento da interatividade e de exploração dos dados espaciais. Ao incorporar a função de interface, na Web, os mapas também tornam mais intuitiva a integração entre os bancos de dados espaciais distribuídos (QUEIROZ FILHO, RODRIGUES, 2007). 


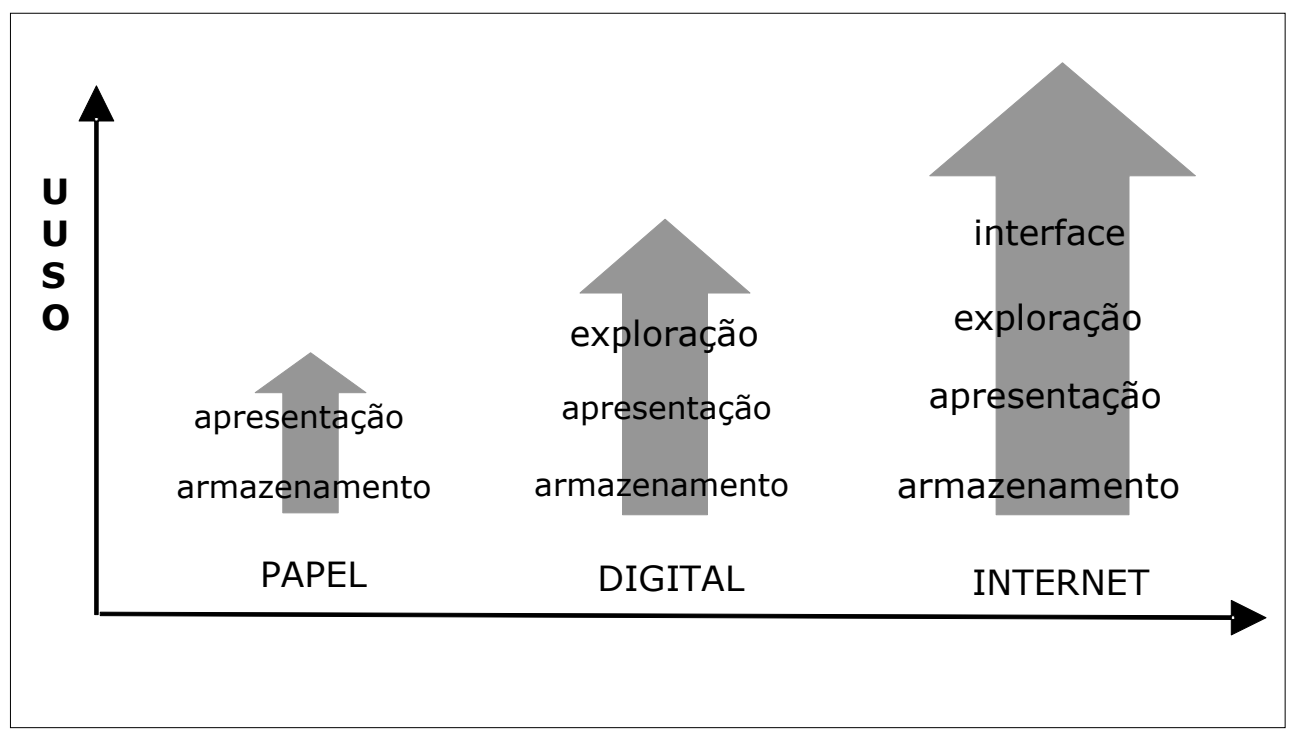

MEIO

Figura 1: Representação esquemática da expansão do uso dos mapas Fonte: Queiroz Filho; Rodrigues (2007)

A integração de mapas via Web foi associada à expressão mash-up. Segundo Butler (2006), mash-up referia-se originalmente à mistura de trilhas musicais e foi apropriado para denominar websites que integravam dados de diferentes. Padronizados, os mapas e as imagens de satélites disponíveis na Web, fornecidos por diferentes agências, podem ser incorporados por outros serviços desse meio. O IBGE, por exemplo, permite a visualização de alguns dados do censo demográfico de 2010 sobre o ambiente Google Maps. O usuário observa figuras geométricas proporcionais do mapa temático sobrepostas às imagens de satélite. Ao clicar sobre os círculos, têm acesso a outras informações estatísticas. O Google fornece as imagens e, o IBGE, os dados do censo (Disponível em: < http://www.censo2010.ibge.gov.br/terrasindigenas/ >. Acesso em: 16 out. 2012).

\section{A integração e o compartilhamento dos mapas na Web}

Na Web, a integração e o compartilhamento de informações são sinônimos da palavra interoperabilidade. Para Goodchild et al (1997), interoperabilidade é a capacidade de compartilhar e trocar informações de um sistema. Seus principais objetivos são:

$\checkmark$ Tornar aberta a estrutura interna dos arquivos de dados;

$\checkmark$ Viabilizar a conversão de dados entre os programas; 
$\checkmark$ Popularizar a interação com o usuário.

A interoperabilidade permite que diversas organizações forneçam dados geográficos via Web, independentemente das suas escolhas tecnológicas, processos de produção, ou cultura interna (DAVIS et al, 2009).

De acordo com Cellarius (1998), a interoperabilidade é a chave para a integração das informações, e deve estar lastreada pelo desenvolvimento de uma infraestrutura de padrões adotados pela comunidade internacional. A interoperabilidade pode ser definida como a capacidade de um sistema ou de seus componentes para compartilhar informações e aplicações, independentemente da sua heterogeneidade (BISHR, 1998). Em decorrência, a interoperabilidade se caracteriza por dois processos distintos: a padronização dos componentes e a sistematização de termos e conceitos, como ilustra a figura 2.

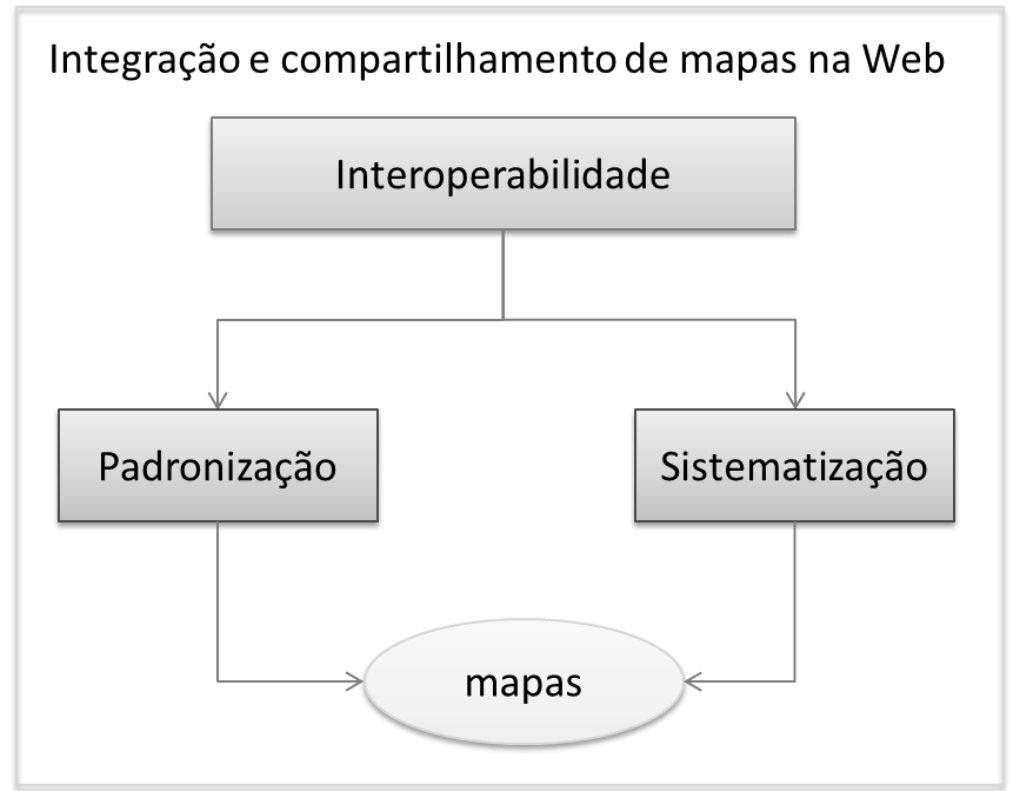

Figura 2: Representação dos processos da interoperabilidade de mapas na Web.

No contexto dos mapas, a padronização extrapola os seus atributos intrínsecos ${ }^{3}$. Como descrito a seguir, a padronização assume distintas denominações, das quais se destacam: metadados e serviços geográficos da Web. A sistematização de conceitos e termos está associada à Web semântica e se manifesta nas pesquisas sobre ontologias.

\footnotetext{
${ }^{3}$ A padronização dos atributos intrínsecos aos mapas, como escala, projeção e coordenadas não foi contemplada nesta pesquisa. Para mais informações sobre a escala no meio digital consultar QUEIROZ FILHO, A.P.; RODRIGUES, M. Arte de voar em mundos virtuais. São Paulo: Annablume. 2007. 162p.
} 


\section{Padronização}

As iniciativas de padronização mais importantes são coordenadas por instituições internacionais. Destacam-se:

$\checkmark \quad$ A International Organizational for Standards - ISO. É o maior colaborador mundial para edição de padrões e normas internacionais. Foi estabelecida em 1947 e conta com uma rede de institutos nacionais de normalização de 163 países (ISO, 2010).

$\checkmark$ O W3C (World Wide Web Consortium), consórcio internacional fundado por Tim Berners Lee, em 1994, que desenvolve padrões para tecnologias para garantir o crescimento da Web em longo prazo (W3C, 2012).

$\checkmark$ O OGC (Open Geospatial Consortium) é uma organização sem fins lucrativos formada por membros da comunidade internacional e voluntários, que desenvolve padrões para dados e serviços baseados em localização espacial. Fundada em 1994, é formada por quatrocentas e duas companhias, agências governamentais e universidades (OGC, 2012).

$\checkmark$ O FGDC (Federal Geographic Data Committee) é um comitê inter-agências que coordena o desenvolvimento, utilização, compartilhamento e disseminação de dados geoespaciais nos Estados Unidos (FGDC, 2012).

Os esforços de padronização relacionados ao compartilhamento de mapas na Web podem ser observados na crescente utilização de metadados e serviços de informações geográficas. Os metadados podem ser definidos como dados sobre os dados (GREEN; BOSSOMAYER, 2002). No caso dos mapas, descrevem o histórico do seu processo de obtenção e produção, o seu conteúdo, sua qualidade, sua condição e demais características.

O grande desafio para a sua criação é estabelecer um padrão compacto e equilibrado para as diferentes necessidades dos usuários, isto é, considerar a melhor forma de representar o seu conteúdo, e, simultaneamente, o custo e o tempo para a sua documentação.

Para Sen (2004), o conceito de metadados evoluiu muito, desde a década de 1960. No início, as discussões tratavam aspectos muito simples, como os nomes dos arquivos e os seus componentes. Posteriormente, a descrição dos dados, os modelos de dados e a incorporação de classes e hierarquias, relacionadas às linguagens de programação orientadas aos objetos. Essas discussões confluíram, no início dos anos 2000, para a internacionalização dos padrões e respectivas formas de intercâmbio. 
Os serviços geográficos viabilizam a disseminação dos mapas em uma magnitude ainda maior pela Web. São aplicações modulares e auto descritivas da Web que permitem a análise e manipulação de dados espaciais (SCHUT, 2007). Seu grande potencial está relacionado à possibilidade de composição, isto é, de formar cadeias de serviços de informações geográficas (LUTZ, 2007).

Existem inúmeros tipos de serviços. De acordo com OGC (2006), o Web Map Service (WMS) produz mapas dinamicamente a partir de informações geográficas disponíveis em servidores remotos. Esse protocolo determina como o cliente deve requisitar as informações georeferenciadas ao servidor e como deverá ser a resposta ao cliente. Esse padrão internacional considera que um mapa não é o dado geográfico em si, mas um "retrato" das informações geográficas, na forma de arquivo digital, adequado para exibição em uma tela de computador, parte das vezes no formato de figura (extensões: PNG, JPEG ou GIF).

Esses serviços podem realizar operações simples como, por exemplo, o cálculo de área de influência (buffer), ou operações complexas, a partir da composição, ou encadeamento, de diversos serviços (FOERSTER et al, 2009). Um dos desafios para composição de serviços de informações geográficas refere-se à integração dessas cadeias de serviços, o que envolve o descobrimento do serviço, a composição e a execução (LEMMENS et al, 2007).

\section{Sistematização de conceitos e termos}

A sistematização de conceitos e termos, presente na figura 2, se expressa pelas denominações Web Semântica e ontologias.

A Web semântica não é uma nova Web, mas sim uma extensão sobre a qual a informação recebe um significado bem definido, permitindo que pessoas e computadores trabalhem em cooperação (BERNERS-LEE et al, 2001).

Para Breitman et al (2007), a expressão Web semântica surgiu em contrapartida à Web sintática. A Web sintática, que caracteriza a Web atual, possui dois atributos principais: 1) excepcional volume de informações e 2) dificuldade de avaliar, classificar e interpretar essa quantidade de informações. A Web semântica pode ser considerada um modelo para organizar as informações da Web, assim como a taxonomia para a Botânica, e viabilizar o uso de computadores nas tarefas de classificação e interpretação dos conteúdos da Web. 
A Web Semântica depende da possibilidade de uso compartilhado de vocabulários, cujo significado seja descrito de uma forma inequívoca e processável por máquina (MASOLO et al, 2003).

A Interoperabilidade semântica pode ser alcançada se os dois agentes, seres humanos e máquinas, concordarem sobre a forma de entender os dados. O nível de compreensão pode ser diferente e depende, principalmente, da complexidade do conhecimento formalizado (MAUÉ et al, 2009).

A ontologia, do ponto de vista da Filosofia, trata do ato de existir. É originária do Grego (onto - ser) e (logia - discurso) e caracteriza as reflexões sobre as coisas que existem (LAURINI, 2007).

No entanto, o termo foi recentemente apropriado por diversas áreas do conhecimento, como a Inteligência Artificial e a Ciência da Computação. Nessas áreas, pode é definido como especificações formais de uma conceituação compartilhada (GRUBER, 1993).

De acordo com Breitman et al (2007), as ontologias são modelos conceituais que capturam e tornam explícito o vocabulário usado em aplicações semânticas. Essas alternativas de organização e formalização do conteúdo da Web em linguagem computacional serão a língua franca da Web semântica.

Para Fensel et al (2003), a ontologia especifica quais conceitos representar e como eles estão inter-relacionados. Relaciona-se a três principais aspectos: 1) definição de conceitos, 2) formalização do conceito em uma linguagem computacional e 3) requisição de que esse conceito seja consensual, isto é, que diferentes pesquisadores estejam de acordo sobre seu significado (FENSEL et al, 2003).

Essa sistematização de termos possui grande importância no compartilhamento e integração de mapas. Um dos exemplos é a sistematização dos nomes dos planos de informações que compõem os mapas. O termo "rio" é simples de ser compreendido e traduzido, mas seus correlatos: arroio, córrego, furo, igarapé, riacho e ribeirão dificultam ou impedem sua interpretação por usuários de outros países e pelas máquinas.

\section{Análise e tendências}

\section{Análise dos mapas na web}


As transformações relacionadas à inserção dos mapas na Web foram apreciadas a partir de quatro principais aspectos: popularização, ampliação de potencial de uso, volatilidade das terminologias e compreensão do conteúdo pelas máquinas.

Com a Web, a popularização dos mapas é incontestável. Se, na época do Renascimento era objeto raro, que circulava somente em altas esferas da nobreza, clero e forças armadas (RAISZ, 1969), atualmente é considerado um documento comum, muitas vezes descartável. Seu uso se multiplicou, mas sua durabilidade e valor histórico se reduziram drasticamente. Em um curto período, a Web se tornou um dos maiores meios de comunicação, e os mapas, um dos seus mais importantes componentes (KRAAK; BRONW, 2001). Do ponto de vista da produção de mapas, a Web suplantou, há muito, a importância da imprensa de Gutenberg. A estimativa de produção mundial, em 1999, era de aproximadamente 10 milhões de mapas por dia. Em 2003, a estimativa indica mais de 20 milhões de mapas gerados diariamente pela Web (PETERSON, 2003). Em 2013, seria razoável projetar uma cifra diária de cerca de 80 milhões de mapas.

Além de se tornar muito mais presente no cotidiano dos cidadãos, seu potencial de uso se ampliou. Da mesma forma que os blocos de montar (ex.: Lego), seus componentes, de formato padronizado, permitem construir diferentes tipos de representação da superfície terrestre. Os mapas podem ser utilizados em diferentes circunstâncias, pois possuem uma estrutura modular que garante a versatilidade necessária para atender um grande espectro de aplicações.

Também é relevante considerar que a importância do mapa se expandiu mesmo que ele não seja visualizado. Em vários sistemas da Web, o mapa representa a base para as operações espaciais. Quando um usuário busca a farmácia mais próxima, por exemplo, a resposta pode ser obtida sem que sua visualização ocorra. Com frequência, adota-se uma hierarquia para atender às necessidades de localização espacial. Os sistemas respondem às consultas, em primeiro lugar, de forma textual - ou por voz -, indicando os endereços, as principais vias de acesso, rotas alternativas e, somente se necessário, fornecem o mapa, a representação cartográfica que forma a base dessa estrutura hierárquica.

Com sua veiculação na Web, nota-se o crescimento do número de terminologias associadas aos mapas. Elas surgem conforme a velocidade do desenvolvimento tecnológico. Estão também relacionadas às estratégias de Marketing de corporações de diversos segmentos. Por essas razões, muitas vezes se tornam efêmeras. 
Nos últimos trinta anos, poucos desses termos se revelaram perenes: Cartografia Multimídia, Cyber Cartografia, Cartografia Automatizada, Cartografia Digital, Geovisualização, Visualização Cartográfica, Web Cartography, Location Based Services, Mobile GIS, Mobile Cartography, Geomática, Geoprocessamento, Sistemas de Informações Geográficas, Web GIS, Internet GIS e Online GIS.

Esse fenômeno também ocorre com os mapas. Eles têm sido associados indistintamente aos dados e/ou informações. Termos como informações espaciais, dados geográficos, dados geo-espaciais, geo-informações, aparecem de forma confusa nos textos da área e muitas vezes são, erroneamente, tratados como sinônimos de mapas.

É pertinente, neste contexto, questionar a durabilidade desses termos tecnológicos. Se considerarmos a tendência volátil das denominações tecnológicas, é plausível ponderar que alguns termos serão substituídos em curto período de tempo, mesmo que sua essência permaneça válida.

Em contrapartida, Wood (2005) defende a ideia de que a essência da Cartografia não se alterou com a ampliação e a diversificação das formas de utilização dos mapas. Para o autor, a Cartografia continua suprema, pois sua história é mais longa e forte do que as tecnologias desenvolvidas para instrumentá-la. Os Sistemas de Informações Geográficas (SIG), os Modelos Digitais de Elevação (MDE), os Sistemas de Gerenciamento de Bancos de Dados (SGBD), entre outros, contribuem muito mais para fortalecê-la do que para diminuir sua influência, pois utilizam ou estão associados às representações cartográficas.

O último aspecto da análise aborda a perspectiva dos mapas serem compreendidos por máquinas. Com o crescimento do volume de informações que circulam na Web e as proporcionais dificuldades de interpretação e classificação do seu conteúdo, emergiu a necessidade de sistematização do vocabulário associado aos mapas. Com a associação unívoca de significado, os programas e máquinas poderão realizar operações muito mais sofisticadas de localização e gerenciamento de informações.

É interessante observar que essa moderna face do desenvolvimento tecnológico, que depende da possibilidade de uso compartilhado de vocabulários, seja frequentemente associado a exemplos muito antigos. Para definir ou explicar a Web semântica e as ontologias, são comuns as referências à árvore de Porfírio, à Torre de Babel, ao esperanto e às taxonomias. 


\title{
Tendências
}

São inúmeras as tendências relacionadas aos mapas na Web. Neste artigo, destacam-se: Infraestruturas de Dados Espaciais, mapas colaborativos, estratégias de localização, digital Earth e computação em nuvem.

Conforme o Federal Geographic Data Committee (FGDC, 2008) uma Infraestrutura de Dados Espaciais (IDE) pode ser definida como:

\begin{abstract}
"As tecnologias, políticas, critérios, padrões e pessoas necessárias para promover o compartilhamento de dados geoespaciais através de todos os níveis de governo, setores privado, órgãos sem fins lucrativos e a academia. Ele fornece uma base ou estrutura de práticas e relacionamentos entre produtores de dados e usuários que facilitam o uso e compartilhamento de dados. É um conjunto de ações e novos modos de acessar, compartilhar e usar dados geográficos que permite tornar mais compreensível a análise do dado para ajudar tomadores de decisões escolherem o melhor curso da ação (FGDC, 2008, p.iv)."
\end{abstract}

Em decorrência, uma IDE não trata somente de questões operacionais, como compartilhamento de dados, mas está diretamente associada às questões jurídicas e políticas, indispensáveis para garantir a funcionalidade desse tipo de estrutura.

Para Nakamura; Queiroz Filho (2012), a importância da IDE está relacionada ao seu potencial de disseminação e uso coletivo das informações geográficas. Colabora para reduzir os problemas de interoperabilidade por intermédio da padronização dos dados. A essência do seu conceito é extremamente simples, mas sua materialização é complexa por causa das diferenças tecnológicas, políticas e culturais das instituições que produzem e utilizam mapas. É possível avaliar que os conjuntos de dados de uma IDE sejam originários do Sistema de Informações Geográficas (SIG) da organização que a constitui. Ainda que a existência do SIG não seja um pré-requisito para o desenvolvimento da IDE, considera-se que o cenário mais usual da sua criação esteja associado à evolução do SIG existente na instituição. Seu principal requisito é atender às próprias demandas, mas deve ser estruturada para suprir às necessidades das IDEs dos níveis superiores da hierarquia (NAKAMURA; QUEIROZ FILHO, 2012).

No caso brasileiro, a IDE foi estabelecida legalmente. A Infraestrutura Nacional de Dados Espaciais (INDE) foi instituída pelo Decreto № 6.666 de 27/11/2008. Seu propósito é catalogar, integrar e harmonizar dados geoespaciais existentes nas instituições do governo brasileiro. Visa ordenar a geração, armazenamento, acesso, compartilhamento, 
disseminação e uso dos dados e evitar a duplicidade de ações e o desperdício de recursos na obtenção de dados geoespaciais das esferas federais, estaduais e municipais de governo.

Os mapas colaborativos representam uma face extremamente interessante da Web. Mesmo que o início das discussões sobre a participação pública e o uso colaborativo de SIG seja de 1995, promovida pelo NCGIA (National Center for Geographic Information and Analysis), sua relevância é crescente e atual. Este evento foi uma das primeiras iniciativas que visava aproximar o público não técnico dos SIGs.

Goodchild (2007) destaca a possibilidade de atuação do cidadão como um sensor voluntário para a produção de mapas. Um dos exemplos é a iniciativa OpenStreetMap, que consiste na construção de um mapa urbano de domínio público, do mundo inteiro, através de um esforço voluntário. Outros conhecidos exemplos de mapas colaborativos são: WikiMapia, Mapufacture, GeoCommons, TerraWiki, FixMyStreet, WholsSick, entre outros.

A diversificação das estratégias de localização é outro aspecto interessante do desenvolvimento tecnológico. Destacam-se os processos denominados como: geotagging, geoparsing e geotargeting (Fu; Sun, 2011).

$\checkmark$ Geotagging: é o processo de adicionar localização (ex:. Latitude/longitude) aos diferentes tipos de arquivos (fotos, vídeos, web sites e rss feeds). É possível associar coordenadas às fotografias de forma que elas sejam associadas aos locais em que foram produzidas;

$\checkmark$ Geoparsing: é o processo de atribuir ou extrair a localização de palavras, frases de um texto ou páginas da web. Diferencia-se de geotagging pelo fato de que palavras ou frases poderem ser associadas a diferentes locais/origens;

$\checkmark$ Geotargeting: é o processo para personalizar o conteúdo de uma página web de acordo com a localização física do usuário/leitor.

A Digital Earth pode ser associada ao discurso de Al Gore, em 1998. O então vice-presidente americano postulava a possibilidade de construir representações tridimensionais do planeta, com múltiplas resoluções, em que pudéssemos incorporar grande quantidade de dados georreferenciados e navegar tanto pelo espaço quanto pelo tempo, para visualizar informações naturais, culturais e políticas sobre o planeta (GORE, 1998). A concepção desse modelo computacional recebeu a denominação de digital earth (FULLER, 1969).

Para Ehlers (2008), a digital earth se baseia no princípio de que qualquer cidadão do planeta, ligado pela internet, poderá acessar livremente um mundo virtual de recursos de informação 
e conhecimento. Foresman (2008) descreve as ferramentas proporcionadas pela digital earth como sendo ubíquas e úteis a todos os cidadãos.

De acordo com Vecchiola et al. (2009), computação em nuvem consiste na junção de computadores de grandes centros de dados, via Web, para proporcionar o ambiente virtual de processamento aos seus clientes. Em outras palavras, computação em nuvens é união de servidores da Web para ampliar a capacidade computacional dos usuários. Para Yanga et al (2011), a ascensão da digital earth, dos mapas colaborativos, das estratégias de localização e dos serviços geográficos depende diretamente da ampliação da capacidade de processamento computacional, pois requerem grandes volumes de dados, acesso simultâneo e complexas análises espaço-temporais.

\section{CONSIDERAÇÕES FINAIS}

O contexto dos mapas na Web foi analisado considerando sua adaptação à comunicação via rede. A inserção dos mapas na Web implicou na necessidade de integração e compartilhamento. Esse binômio pode ser traduzido por interoperabilidade, que está relacionada aos processos de padronização de dados e a sistematização de conceitos e termos. Na Web, o volume de mapas produzidos aumentou exponencialmente e suas funcionalidades se ampliaram. No entanto, parte dos termos relacionados aos mapas se tornou efêmera dada as constantes inovações oriundas do desenvolvimento tecnológico. Um dos desafios atuais é tornar o conteúdo dos mapas globalmente compreensível, por homens e máquinas. As Infraestruturas de Dados Espaciais - IDE, os mapas colaborativos, a diversificação das estratégias de localização, a digital Earth e a computação em nuvens revelam significativas tendências de desenvolvimento do momento.

Com o mapa na Web, observa-se que houve um desproporcional aumento da importância da informática. Nesse período de adaptação, o meio parece ter mais destaque do que a própria representação cartográfica. Com a padronização, os mapas e respectivos componentes estão mais claramente dissociados, assim como sua produção. O mapa em si torna-se um instante de visualização dos dados geográficos, gerado por protocolos padronizados de comunicação.

Por outro lado, a difusão de programas gratuitos e o alto nível das suas interfaces permitem a realização de complexas tarefas com um diminuto conhecimento de informática. Além 
disso, destaca-se também potencialidade da colaboração voluntária na produção de mapas. Iniciativas que promovam a motivação dos usuários e que garantam a qualidade desses dados certamente transformarão o cenário dos mapas dos próximos trinta anos.

\section{REFERÊNCIAS BIBLIOGRÁFICAS}

AGARWAL P. Ontological considerations in GIScience. International Journal of Geographic Information Science, v. 19, n. 5, p. 501-536, 2005.

ANDREWS, J.H. What Was a Map? The Lexicographers Reply. Cartographica, vol. 33, no. 4, p.1-11. 1996.

BERNERS-LEE, T.; HANDLER, J.; LASSILA, O. The semantic web: A new form of web content that is meaningful to computers will unleash a revolution of new possibilities. Scientific American, v. 284, n. 5, p. 34-43. 2001.

BISHR, Y. Overcoming the Semantic and Other Barriers to GIS Interoperability. International Journal of Geographical Information Science, v. 12, n. 4, p. 299-314, jan. 1998.

BREITMAN, K.K; CASANOVA, M.A.; TRUSZKOWSKI, W. Semantic web: concepts, technologies and applications. New York: Springer-Verlag. 2007. 327p.

BUTLER, D. Mashups mix data into global service. Nature, v. 439, n. 5, p. 6-7, jan, 2006.

CELLARIUS, P. Interoperability: the key to competitive supply in the Global Information Society GIS. Computer Standards \& Interfaces. v. 20, 1998, p. 123-128.

DAVIS JR. C. A.; FONSECA, F. T.; CÂMARA, G. Beyond SDI: Integrating Science and Communities to Create Environmental Policies for the Sustainability of the Amazon. International Journal of Spatial Data Infrastructures Research. 2009. v. 4, p. 156-174.

EHLERS, M. Geoinformatics and digital earth initiatives: a German perspective. International Journal of Digital Earth. 2008. v. 1, n. 1, p. 17-30.

FENSEL, D.; HENDLER, J.; LIEBERMAN, H.; WAHLSTER,W. Spinning the Semantic Web. Cambridge, MA: MIT Press. 2003. 
FGDC- Federal Geographic Data Committee. Geographic Information Framework Data

Content Standard. 2008. Disponível em: <http://www.fgdc.gov/standards/projects/FGDCstandards-projects/framework-data-

standard/GI_FrameworkDataStandard_Part0_Base.pdf>. Acesso em: 05 out. 2012.

FGDC (Federal Geographic Data Committee). Recursos e atividades desenvolvidas. Disponível em: <http://www.fgdc.gov/>. Acesso em: 10 mar. 2012.

FOERSTER, T., BROERING, A., JIRKA, S., MUELLER, J. Sensor Web and Geoprocessing Services for Pervasive Advertising. In: Workshop for pervasive advertising, Luebeck, Alemanha. 2009.

FORESMAN, T. W. Evolution and implementation of the Digital Earth vision, technology and society. International Journal of Digital Earth. 2008. v.1, n. 1, p. 4-16.

FU, P.; SUN, J. Web Gis: principles and applications. ESRI Press: California. 2011, 296p.

FULLER, 1969, Operating Manual for Spaceship Earth. Touchstone. New York. Disponível em: http://classes.design.ucla.edu/Spring07/9-1/_pdf/3-fuller_operating-manual.pdf. Acesso em: 17 out 2012

GOODCHILD, M.; EGENHOFER, M.J.; FEGEAS, R. Interoperating GISs: Panel on computational implementations of geographic concepts. In: INTERNATIONAL CONFERENCE \& WORKSHOP ON INTEROPERATING GEOGRAPHIC INFORMATION SYSTEMS. Santa Bárbara, 1997. Anais eletrônicos.

Disponível

em: <http://www.ncgia.ucsb.edu/conf/interop97/report.html\#section1 >. Acesso em: 10 out. 2012.

GORE, A. The Digital Earth: Understanding our planet in the 21st Century. Disponível em: <. http://www.isde5.org/al_gore_speech.htm>. 1998. Acesso em 24 out 2012.

GREEN, D.; BOSSOMAIER, T.; Online GIS and Spatial metadata. New York: Taylor \& Francis. 2002. $222 \mathrm{p}$.

GRUBER, T.R. A translation approach to portable ontologies. Knowledge Acquisition, v. 5, p. 199-220, 1993. 
GUARINO, N. Formal ontology, conceptual analysis and knowledge representation. International Journal of Human-Computer Studies, v. 43, p. 625-640. 1995.

INTERNATIONAL CARTOGRAPHIC ASSOCIATION, Multilingual Dictionary of Technical Terms in Cartography. Steiner: Wiesbaden. 1973.

ISO (International Organization for Standardization). Recursos e atividades. Disponível em: <http://www.iso.org/>. Acesso em: 10 mar. 2012.

KRAAK, M.; BROWN, A. Web Cartography: developments and prospects. London: Taylor and Francis. 2001, 213p.

KUHN, W. Geospatial Semantics: Why, of What, and How? Journal of Data Semantics. v. 3534. p.1-24. 2005.

LAURINI, R. Pre-consensus Ontologies and Urban Databases. In: Ontologies for Urban Development, Edited by Jacques Teller, John R. Lee and Catherine Roussey, Springer Verlag, Studies in Computational Intelligence, v. 61, pp. 27-36, 2007.

LEMMENS, R.L.G., GOULD, R.A., WYTZISK, A. Enhancing geo - service chaining through deep service descriptions. Transactions in GIS. 2007. v. 11, n. 6, p. 849-871.

LUTZ, M. Ontology-based descriptions for semantic discovery and composition of geoprocessing services. Geoinformatica. 2007. v. 11, p. 1-36.

LYNCH, C. Big data: How do your data grow? Nature 455, 2008, p.28-29 doi:10.1038/455028a

MACEACHREN, A.M.; KRAAK, M.J. Research challenges in geovisualization. Cartography and Geographic Information Science, v.28, n.1, 3-12. 2001.

MASOLO, C.; BORGO, S.; GANGEMI, A.; GUARINO, N.; OLTRAMARI A.; SCHNEIDER, L. DOLCE: a Descriptive Ontology for Linguistic and Cognitive Engineering. WonderWeb Project, Deliverable D17 v2.1, 2003.

MAUÉ, P. An extensible semantic catalogue for geospatial web services. International Journal of Spatial Data Infrastructures Research. 2008. v. 3, p. 168-191. 
MONMONIER, M.S. Technological transition in cartography. Wisconsin: University Wisconsin Press. 1985. 282 p.

MUEHRCKE, P.C.; MUEHRCKE, J.O.; KIMERLING, A.J. Map use: reading, analysis and interpretation. Madison: JP Publications. 2001. 647p.

NAKAMURA, E.T.; QUEIROZ FILHO, A.P. Infraestrutura de dados espaciais: exemplo do Parque Estadual de Intervales - SP. Revista Brasileira de Cartografia v.64 n. 3, 2012, p. $22-$ 38.

OGC - OpenGIS Consortium. The Abstract Specifications. Open GIS Consortium Technical Committee, 2006. Disponível em: <http://www.opengeospatial.org/standards/as >. Acesso em: 01 out 2012.

OGC (Open Geospatial Consortium). Recursos e atividades desenvolvidas. Disponível em: < http://www.opengeospatial.org/>. Acesso em: 10 mar. 2012.

PENG, Z.R.;TSOU, M.H. Internet GIS: distributed Geographic Information Services for the Internet and wireless networks. New Jersey: John Wiley and Sons. 2003. 679p.

PETERSON, M. P. Maps and the Internet. Hungary: Elsevier. 2003. 451p.

PETERSON, M. P. Trends in Internet map use: a second look. In: INTERNATIONAL CARTOGRAPHIC ASSOCIATION. Anais eletrônicos do ICA 99. Disponível em: <http://maps.unomaha.edu/MP/articles/ICA99/trends99.html>. Acesso em: 21 out. 2012.

QUEIROZ FILHO, A.P.; RODRIGUES, M. Arte de voar em mundos virtuais. São Paulo: Annablume. 2007. 162p.

RAISZ, E. Cartografia Geral. Rio de Janeiro: Científica. 1969. 414 p.

ROBINSON, A.H.;MORRISON, J.L.; MUEHRCKE, P.C.;KIMERLING, A.J.; GUPTILL, S.C. Elements of cartography. Danvers: John Willey \& Sons. 1995. 674p.

SEN, A. Metadata management: past, present and future. Decision Support Systems v.37, 2004, p. 151- 173. 
VECCHIOLA, C.; CHU, X.; BUYY, R. Aneka: A Software Platform for.NETbased Cloud Computing. Melbourne. 2009.

W3C (Word Wide Web Consortium). Recursos e atividades desenvolvidas. Disponível em: <http://www.w3.org/>. Acesso em: 10 mar. 2013.

WOOD, M. Cartography is still supreme. In: INTERNATIONAL CARTOGRAPHIC CONFERENCE, 2005, La Coruña. Proceedings of International Cartographic Conference. La Coruña: ICC, 2005. 1 CD-ROM.

YANGA, C.; GOODCHILD, M.,HUANGA, Q.; NEBERTC, D. ; RASKIND, R.; XUE, Y.; BAMBACUSF, M.; FAYE, D. Spatial cloud computing: how can the geospatial sciences use and help shape cloud computing? International Journal of Digital Earth. 2011. v. 4, n. 4, p.305-329. 